Etnográfica

Revista do Centro em Rede de Investigação em

Antropologia

vol. 21 (1) | 2017

Vol. 21 (1)

\title{
Nosotros también somos indígenas: la vulnerabilidad del naturalismo en contextos occidentales de convivencia entre especies
}

We are also indigenous: the vulnerability of naturalism in western contexts of interspecies coexistence

\section{Santiago M. Cruzada}

\section{(2) OpenEdition}

\section{Journals}

Edición electrónica

URL: https://journals.openedition.org/etnografica/4810

DOI: 10.4000/etnografica.4810

ISSN: 2182-2891

\section{Editor}

Centro em Rede de Investigação em Antropologia

Edición impresa

Fecha de publicación: 1 febrero 2017

Paginación: 49-71

ISSN: 0873-6561

Referencia electrónica

Santiago M. Cruzada, «Nosotros también somos indígenas: la vulnerabilidad del naturalismo en contextos occidentales de convivencia entre especies», Etnográfica [En línea], vol. 21 (1) | 2017, Publicado el 11 marzo 2017, consultado el 09 febrero 2022. URL: http://journals.openedition.org/ etnografica/4810 ; DOI: https://doi.org/10.4000/etnografica.4810

\section{(c) () 8}

Etnográfica is licensed under a Creative Commons Attribution-NonCommercial 4.0 International License. 


\title{
Nosotros también somos indígenas: la vulnerabilidad del naturalismo en contextos occidentales de convivencia entre especies
}

\author{
Santiago M. Cruzada
}

Muchos de los estudios ambientales en la disciplina antropológica asumen que el naturalismo es una ontología característica de la sociedad occidental a través de la cual se dibuja una frontera entre la naturaleza y la sociedad o que separa a los seres humanos de los animales. Este modelo teorético se fundamenta al concebir una naturaleza compartida, unitaria y ajena a la voluntad humana, sobre la que se aplican diversas formas culturales de entenderla. Por contraposición se han configurado paradigmas monistas de relaciones ecológicas en otras partes del mundo, sirviendo el naturalismo como base para erigir explicaciones alternativas acerca de cómo sociedades no occidentales se relacionan con el entorno. Sin embargo, esta asignación cosmológica enclaustra la reflexión socio-ambiental en Occidente, reforzando la disyunción entre occidental y no occidental, al mismo tiempo que encierra el análisis de una posible generación creativa de mundos no dicotómicos en sociedades occidentales. Este artículo problematiza esas asunciones a través de un ejemplo extraído del trabajo de campo en el suroeste de Extremadura (España), estableciendo un punto de partida teórico para el debate bajo la idea de una "antropología de la convivencia”. Esta abarca y comprende etnográficamente las relaciones entre diferentes seres que habitan el entorno más allá de una circunscripción estereotipada contextual.

PALABRAS-CLAVE: epistemología, medio ambiente, naturalismo, ontología, etnografía, convivencia entre especies.

We are also indigenous: the vulnerability of naturalism in western contexts of interspecies coexistence - Many environmental studies in the anthropological discipline assume that naturalism is a characteristic ontology of western society through which a border is drawn between nature and society or that separates human beings from animals. This theoretical model is based on the conception of a shared nature, unified and unrelated to the human will, on which different cultural forms of understanding it are applied. In contrast, monistic paradigms of ecological relations have been set up in other parts of the world, serving the naturalism as a basis for constructing alternative explanations of how non-western societies relate to the environment. However, such a cosmological assignment entails a socio-environmental reflection in the West, reinforcing the western/non-western disjunction while enclosing the analysis of the creative generation of non-dichotomous worlds in western societies. This article problematizes these assumptions through an example from the fieldwork in the southwest of Extremadura (Spain), establishing a theoretical starting point for the debate under the idea of an "anthropology 


\begin{abstract}
of coexistence" that ethnographically encompasses and comprehends the relationships among different beings that inhabit the environment beyond a stereotyped contextual circumscription.
\end{abstract}

KEYWORDS: epistemology, environment, naturalism, ontology, ethnography, interspecies coexistence.

CRUZADA, Santiago M. (smoncru@upo.es) - Grupo de Investigación Social y Acción Participativa (GISAP), Departamento de Antropología Social, Psicología Básica y Salud Pública, Universidad Pablo de Olavide, España.

\title{
INTRODUCCIÓN
}

La historia teórica de las relaciones entre los seres humanos y la naturaleza consolida la idea de que los modelos binarios sobre el cosmos se agudizaron en Occidente a partir del pensamiento cartesiano y mecanicista (Glacken 1996). ${ }^{1}$ Una nueva ciencia moderna positiva, más eficiente en la descripción de la realidad y con reminiscencias platónicas y judeocristianas, fue la base para presentar a los humanos por encima del resto de seres (Ingold 1994). Más tarde, con el surgimiento ilustrado, se incidió en las dicotomías sobre la propia existencia: mente-cuerpo, individuo-sociedad o naturaleza-cultura son un ejemplo (Sahlins 2011). Esto fue el sustrato, a grandes rasgos, para el esquema de pensamiento que hoy prevalece entre nosotros. El programa dicotómico nos ha hecho herederos de una concepción particular de un mundo jerarquizado, regulado por oposiciones binarias, que ha sido denominado naturalismo occidental (Descola 2001).

Sin embargo, ¿es posible entender formas y modos de vida occidentales más allá de este naturalismo histórico, teórico y social? En este artículo pretendo discutir la asignación del modelo teorético naturalista al contexto occidental basado en presupuestos dicotómicos que separan la naturaleza de la sociedad. Argumento que su formulación teórica esencializa territorios, culturas y perspectivas teóricas bajo un homogéneo paraguas cosmológico y analítico (Ramos 2012; Bessire y Bond 2014; Vigh y Sausdal 2014; Guillo y Hamilton 2015), reduciendo las condiciones de posibilidad para analizar la generación creativa de mundos no dicotómicos en sociedades occidentales (Candea y Alcayna-

l Este texto ha sido elaborado en el marco de financiación de las Ayudas para la Formación del Profesorado Universitario (FPU, 2013) del Ministerio de Educación, Cultura y Deporte del Gobierno de España. Agradezco a la Comisión Editorial de Etnográfica y a los revisores anónimos de la revista sus comentarios y críticas constructivas. Asimismo manifiesto mi deuda a colegas del departamento con los que he debatido y a la gente de Segura de León que me abrió la puerta de sus casas. 
-Stevens 2012; Ingold 2016). Si bien entiendo los avances logrados en la teoría social que por contraposición al naturalismo ha forjado grandes campos de investigación en contextos no occidentales, quiero enfatizar en la idea de que en Occidente muchos de nosotros también (o todavía) somos "indígenas".

Dividiré el texto en tres apartados. En el primero repasaré cómo se construyen modelos teoréticos con base al naturalismo y cómo, al mismo tiempo, se crea un "otro" ecológico que cuestiona el universo occidental. En el siguiente epígrafe esbozaré algunas críticas sobre esos postulados. La última parte del texto estará dedicada a la composición teórica que deseo presentar como desembocadura del debate y que lleva como rúbrica una "antropología de la convivencia”, la cual intentará exceder lo que considero es la parcialidad de las teorías socio-ambientales contemporáneas a partir de un ejemplo extraído de mi trabajo de campo centrado en las relaciones entre humanos y animales.

\section{EL NATURALISMO OCCIDENTAL}

\section{Y LA CREACIÓN DEL “OTRO” ECOLÓGICO}

El programa dicotómico propició que durante las tres últimas décadas los estudios antropológicos centrados en ecología se propusieran la deconstrucción misma del concepto de "naturaleza". Esta no era única, cambiaba dependiendo de prácticas, circunstancias y lugares en las que se representase o percibiese, y opuesta a la "sociedad" mostraba precariedad para los análisis socio-ambientales. Sin embargo, las investigaciones no se realizaron con tanta asiduidad ni con marcos tan deconstructivos donde el naturalismo operaba, es decir, en Occidente, dando por hecho que la dicotomía sigue existiendo como forma moderna dominante de pensamiento (Escobar 2000: 118).

Una primera aproximación que sentó las bases comenzó a realizarse en la década de los 90 observando cómo existían cosmologías a lo largo del planeta que no contemplaban una disyuntiva radical entre naturaleza y cultura, o entre humanos y animales, como la que se producía en contextos occidentales desde donde los investigadores partían (Croll y Parkin 1992; Gray 1996). Superar los prejuicios que los investigadores occidentales llevaban al campo en el estudio de sociedades no occidentales sería, desde entonces, una meta primordial (Escobar 2000; Henare, Holbraad y Wastell 2007).

Estos planteamientos de partida han sido etiquetados como "modos de identificación naturalista" (Descola 2001, 2004, 2012) y se insertan en un marco cosmológico mayor denominado naturalismo. Su definición paradigmática la ofrece Descola, abriendo un campo idóneo para la construcción de postulados monistas. El naturalismo, dice el autor, es la creencia de que la naturaleza existe gracias a un principio ajeno tanto a la suerte como a la voluntad humana, siendo típico de cosmologías occidentales en las que se crea un dominio ontológico específico donde nada ocurre sin una razón o una causa, 
y llega a ser una presuposición "natural” epistemológica e incrustada en nuestro sentido común (Descola 2001: 108-109).

Según Descola (2012) los procesos cognoscitivos universales en los seres humanos se caracterizan por la conciencia de una dualidad de planos entre los procesos materiales, a los cuales denomina "fisicalidad", y los estados mentales, a los que llama "interioridad". El naturalismo compromete la suposición de que las exterioridades de los seres - humanos y no humanos - son similares, mientras que la interioridad sólo es poseída por humanos. Es decir, una naturaleza unitaria sobre la que se superponen muchas culturas o visiones del mundo particulares, o en otras palabras, una naturaleza como objeto externo a nuestro ser subjetivo concebible (Kohn 2015: 317). A él se opondrían otros modelos ontológicos como el animismo, el totemismo o el analogismo, donde las fisicalidades y las exterioridades variarán con respecto al naturalismo. ${ }^{2}$

Para Descola (2001: 106) la forma en que las personas construyen representaciones de su medio ambiente físico y social está organizada a través de patrones significativos y diagramas cognitivos. A partir de ellos se pueden descubrir modelos ontológicos generales en las diferentes relaciones socioecológicas (Descola 2001: 107), teniendo siempre en cuenta que la forma genuina occidental de interaccionar con el medio se basa en un modelo ontológico de "protección" o bien "predatorio". En ambas existe un interés explícito por beneficiarse de la naturaleza y en ellas se ejemplifican de manera clara las pautas mecánico-cartesianas de representación de lo natural (Descola 2001: 110-112).

En tono similar discute Viveiros de Castro los términos en que las epistemologías occidentales ponen en marcha un mecanismo ontológico dicotómico derivado de un programa ilustrado que separa esferas irreductibles en otros contextos (Castro 2004 y 2010). Por ejemplo, argumenta que las oposiciones binarias deberían ser redistribuidas y asociadas en contextos amerindios donde, a diferencia de nosotros los occidentales, residen cualidades de perspectivas relativas y puntos de vista diferentes para los distintos seres que habitan el cosmos (Castro 2004: 37). El naturalismo occidental (para Viveiros de Castro, "multiculturalismo") viene predeterminado por la idea de que la naturaleza existe debido a la universalidad objetiva de los cuerpos y las sustancias, al mismo tiempo que la diversidad cultural está garantizada por la particularidad subjetiva de los espíritus y el significado - una naturaleza universal, muchas

2 Esta concepción está vinculada, como veremos, a un conjunto de argumentos que de manera genérica oponen el naturalismo a formas alternativas de relacionamiento ecológico en otras partes del mundo, pero también a estudios de ciencia, tecnología y medicina, tanto dentro como fuera de la antropología (Candea y Alcayna-Stevens 2012; Latour 2013). A la gama de investigaciones que se focalizan en el análisis de las diversas formas de concebir el ser, la otredad y la realidad como condición para la existencia se les ha venido caracterizando dentro de un movimiento mayor denominado "giro ontológico" (Henare, Holbraad y Wastell 2007; Costa y Fausto 2010; Salmond 2014). 
culturas particulares. Este se opone al "multinaturalismo" (para Viveiros de Castro, cosmologías no occidentales), definido por la unidad del significado y la diversidad de los cuerpos - una cultura universal, muchas naturalezas particulares (Castro 2004: 38).

Marilyn Strathern (2004) o Bruno Latour (2007) se posicionan en este sentido criticando el típico pensamiento popular y académico contemporáneo euro-americano que nos sobrepasa y al mismo tiempo nos es transcendente separando dominios inconmensurables en otros contextos. La idea de multiplicidad es central en sus propuestas alegando que las relaciones son rizomáticas en la vida social y en las interacciones que la gente mantiene con su medio en otros contextos no occidentales, lo cual facilita pensar sin oposiciones y superar las reflexiones que separan a los individuos de las sociedades, de la naturaleza o de los animales. La condición de la modernidad, para Latour (2013), ya no sólo corre un tupido velo que invisibiliza entidades "naturculturales" (Haraway 2003), sino que es bajo esa condición que separa la naturaleza de la cultura con lo que se amenaza la pluralidad de modos de ser (Kohn 2015: 322).

El modelo naturalista aparece definido y contestado en muchos trabajos que se enmarcan dentro de este giro epistemológico de apertura hacia la ontología (Candea y Alcayna-Stevens 2012; De la Cadena 2014). En todos ellos el naturalismo soporta unas críticas que albergan como telón de fondo la idea de que los humanos son los dueños de la naturaleza y que su dominación tiene una localización occidental. En cambio, no se cuestiona qué es la naturaleza en Occidente, cómo se relacionan los humanos con ella en todo Occidente o qué se entiende por Occidente.

Tim Ingold tampoco escapa de construir ideas opuestas a los inconvenientes que la cosmovisión occidental ha incrustado en los análisis sociales. Ingold (1991: 365) cree que el discurso occidental separa ontológicamente los ámbitos de lo subjetivo - mente y significado - y lo objetivo - materia y sustancia. El paradigma occidental está vinculado a las nociones de orden, racionalidad y uniformización, un modelo hilomórfico que concibe la separación diferencial entre formas - comportamiento humano - y sustancias - materias del mundo natural (Ingold 2008: 7). Su semejanza a las ideas anteriormente expuestas es más que evidente, aunque añade la utilización de la noción persona-organismo como hito que supera la linealidad y la estructura jerárquica de las relaciones bio-psico-sociales de la ortodoxia científica occidental (Ingold 2008: 12). Por tanto, sólo cuando el mundo se presenta como algo exterior a lo humano puede ser concebido como "naturaleza", y esa serie proposicional ha sido comúnmente establecida en el mundo occidental, siendo poco realista en otros contextos (Ingold 2000: 40-61). ${ }^{3}$

3 A pesar de ello en un reciente trabajo de revisión al naturalismo que propone Descola, Tim Ingold se retracta sugiriendo que el modelo hilomórfico de producción, como la imposición del diseño [continua] 
La tarea de presentar diversamente el mundo occidental no es fácil, más cuando se tiene la hipótesis de que el modelo naturalista funciona como mecanismo de acción práctica ab initio y como elemento articulador del pensamiento ad aeternum. Menos cuando al mismo tiempo esta creencia sirve como antítesis teórica y como valija donde arrojar las causas de las crisis planetarias medioambientales. El naturalismo se ha convertido en una idea "generalizante" y generalizada, ya sea aquí o allende los mares, estableciendo a su vez un pensamiento diferenciado entre el "Norte" y el "Sur".

Arturo Escobar, por ejemplo, define al modelo naturalista como "colonialidad de la naturaleza", presentando las características de las que se componen este tipo de discursos y prácticas que tienen resonancia desde la Europa post renacentista: clasifica en jerarquías dejando a la naturaleza y a los primitivos al final de la escala; esencializa la naturaleza como fuera del dominio humano; subordina el cuerpo y la naturaleza a la mente; hace subalternas las articulaciones entre el ser, el conocer y el hacer, etc. (Escobar 2011: 51). Todas estas dimensiones del modelo son enseñadas a la mayoría de las personas de Occidente, argumenta, aprendiendo aquellas a desear y depender de un "logocentrismo occidental" que permite pensar la realidad social de manera ordenada y racional (Escobar 2011: 64). En este sentido se posiciona Blaser (2009) al definir su propuesta de ontología política que intenta trascender las premisas de la modernidad eurocéntrica presentadas como universales, cuando simplemente es una más de muchas, o más bien hegemónica entre muchas.

El naturalismo hace su aparición en los diferentes modelos teóricos que tienen como intención desintegrar la herencia de la modernidad occidental, tanto en su plano teórico como social. Aquí hemos presentado algunas de las consideraciones más conspicuas para comprobar cómo en ellas el modelo naturalista ha servido de soporte sobre el que construir paradigmas que dieran rigurosa cuenta de las relaciones que se producen entre los seres humanos y el medio en otras partes del mundo. Pero paralelamente en esa oposición se ha creado un "otro" ecológico que se enfrenta de manera trasversal al "nosotros" occidental, siendo lo primero diverso y lo segundo invariable. El naturalismo ha llegado a ser, como señalan Candea y Alcayna-Stevens (2012: 37), un exótico y desconocido "otro" antropológico en el seno mismo de nuestro "yo" imaginado, que se presenta como un horizonte lejano y como punto de fuga de argumentos antropológicos de otra cosa.

Poco obstáculo pondré a estas desarticulaciones. Las consecuencias devastadoras del naturalismo para el medio ambiente se patrocinan bajo una lógica mecánica, racional, progresista y totalizante del mundo, minando saberes y prácticas en cosmologías locales históricas (Sahlins 2011). Sin embargo, 
entiendo que el naturalismo no sólo puede ser explicado por su propia historia sin necesidad de recurrir a una constatación real y contemporánea de su existencia en ese "homogéneo Occidente": ¿es extrapolable el modelo naturalista a todo Occidente? ¿Hay variabilidad de modelos teoréticos y prácticos en Occidente? ¿En qué medida afecta el naturalismo a saberes y prácticas históricas propias de contextos occidentales?

\section{PROBLEMATIZACIÓN Y VULNERABILIDAD DE LOS POSTULADOS CON BASE EN EL NATURALISMO}

Si bien la deconstrucción del proyecto dualista favoreció ir más allá de los estados y las sustancias para centrarse en los procesos y las relaciones (Descola y Pálsson 2001: 23), han surgido nuevas problemáticas en relación a la correspondencia entre las diferentes concepciones de naturaleza o en la comprobación de cada construcción cultural única de naturaleza, incluida la nuestra occidental (Ellen 2001: 125). En otras palabras, se trata de mirar si efectivamente la naturaleza se construye y negocia sin ambigüedades en el seno de una sociedad.

Sin embargo, la falla en estas nuevas problemáticas es, a mi juicio, que preexiste la idea de que la construcción de la naturaleza en Occidente está bien establecida, sin posibilidad de variaciones, ambigüedades o combinaciones que hacen, efectivamente, afirmar su existencia. Por lo tanto, a priori existe una homogeneización sobre la representación de la construcción social de la naturaleza en Occidente que invalida cualquier tipo de hibridación o coexistencia cosmológica y cultural. De la misma forma se bloquea una posible reflexión sobre la misma porque un modelo naturalista híbrido está condenado a permanecer en el terreno de la utopía o sería, simplemente, una antinomia, una paradoja o un sinsentido, como apunta Descola (2001: 115-119).

El naturalismo se concibe como ontología totalizante, pero no sólo para la esfera académica, sino también para el resto de la sociedad occidental, sin saberse muy bien donde se posiciona realmente (Fischer 2014). Quizás esta asociación intermitente se produzca del no discernimiento de los tipos de naturalismo definidos epistemológicamente o, en el peor de los casos, de su desconocimiento. Diéguez Lucena (2014) señala varios tipos: naturalismo metodológico, naturalismo ontológico, naturalismo epistemológico y naturalismo filosófico. Este último podría englobar indistintamente a los anteriores y, de manera extensa, al que me estoy refiriendo en este texto.

Por otro lado, las teorías con base en el naturalismo suelen asimismo generalizar la idea del indígena como el "otro" ecológico, siendo difícil comprender cómo esas poblaciones, globalizadas o no, nos representan a "nosotros" los occidentales (Morales Inga 2014: 22; Citro y Gómez 2013: 262). Ampliar sin matices una cosmovisión no occidentalizada de algunas sociedades entraña 
el riesgo de difuminar de un plumazo las transformaciones políticas, económicas o históricas de otros muchos grupos sociales de naturaleza diferente dentro de la misma sociedad o territorio (Ramos 2012; Vigh y Sausdal 2014; Kohn 2015), haciendo de casos particulares una totalidad que no tiene por qué tener su correspondencia etnográfica (Bartolomé 2014; Bessire y Bond 2014; Reynoso 2015). Ampliar sin matices una cosmovisión occidentalizada de un gran territorio conlleva un enclaustramiento de los cuestionamientos particulares (Sánchez-Criado 2009: 151) al quedar agotados bajo posiciones herméticas de cualquier idea de contínuum ontológico. Más adecuado sería posicionar a esta de forma "privilegiada" entre otras (Sahlins 2014: 288).

Los resultados que desprenden estos postulados son uniformes y generales con respecto a la cosmología binaria que se supone existe para todo Occidente (Ramos 2012). Si bien es verdad que algunos autores dejan apertura a interacciones polifónicas y matizaciones (Ellen 2001), se presenta como un paradigma con regímenes limitados en el tiempo y en el espacio (Carrithers et al. 2010). La preconcepción de un modelo teorético dicotómico confina la atención que se necesitaría para observar cómo se producen las relaciones entre los seres humanos y los entornos en aquellos lugares donde se supone que la naturaleza se encuentra cosificada y realmente ajena a los humanos (Candea y Alcayna-Stevens 2012).

Más allá de esto, las afirmaciones vertidas sobre nosotros los occidentales oscurecen de forma inmediata la práctica común que se tiene en estos contextos de dotar de "cuerpo social" y de "personalidad humana" a plantas, animales y cosas (Sahlins 2014: 288-289), al mismo tiempo que niega la existencia de experiencias occidentales modernas de animismo o perspectivismo en los procesos socio-subjetivos de organización de la vivencia en el entorno (Pazos 2007: 372; Pedersen 2011). Un ejemplo contradictorio sería el desarrollo de religiones y creencias occidentales que han pervivido a la supuesta imposición de un modelo cartesiano que las domine (Citro y Gómez 2013: 263-264; Bartolomé 2014: 17).

Todo ello redunda en la asunción de que existe un "pensamiento indígena" diferenciado de un homogéneo "pensamiento occidental" (Candea 2011; Bartolomé 2014: 10). Muchas veces se ponen de relieve trabajos abstractos que esencializan a los pueblos indígenas bajo premisas exóticas que no encuentran su paralelo empírico, reforzando desde otros postulados estereótipos sobre esas sociedades ya superados anteriormente, o simplemente posicionándolos en lugares que ellos mismos no han elegido (Pazos 2007; Graber 2015).

Otra cuestión que vulnera los postulados con base en el naturalismo es el rezumo etnocéntrico que muchos trabajos desprenden. La incapacidad de volver a mirar el contexto de partida naturalista hace de estas perspectivas fórmulas paradigmáticas unidimensionales, asemejándose a las antropologías coloniales que olvidaban trabajar con los condicionantes globales más amplios 
(Bessire y Bond 2014; Hage 2014). Existe una ilusión de que las culturas donde el naturalismo no opera han estado aisladas de influencias externas (Marcus y Fisher 2000), lo que define una línea que se desplaza desde "cosmologías no modernas" a "cosmologías modernas", siendo los etnógrafos los conectores entre dos mundos (Sánchez-Criado 2009: 151; Bartolomé 2014: 13; Morales Inga 2014: 31-32).

Todas esas perspectivas repasadas en el anterior epígrafe podrían ser denominadas, sin lugar a dudas, corrientes naturalistas, porque efectivamente desde ellas se afirma que el naturalismo opera en Occidente. En definitiva, son modelos explicativos metonímicos, pues toman de manera precisa la parte - prejuicios de la investigación "occidentalizada" u ontologías supuestamente occidentales - por un todo - sociedad occidental -, mezclando indiscriminadamente epistemología y realidad, coincidiendo en muchas ocasiones pero errando en otras, y finalmente opacando el análisis de una posible generación creativa de mundos no dicotómicos en sociedades occidentales. Esto ha provocado, no obstante, que hayan terminado siendo modelos teoréticos incuestionados y ecuménicos que muestran a la sociedad occidental como la antítesis de la naturaleza o el medio ambiente. Según Guillo y Hamilton (2015: 123-124) lo que se trata no es tanto de garantizar si el naturalismo es verdadero o falso como de determinar los diversos significados específicos que pueda tener para examinarlos con suficiente precisión, ya que no se puede pensar en una imposición automática a todo Occidente de una constitución moderna.

Un proceso reflexivo donde se discutiera la efectividad del naturalismo en contextos occidentales sería saludable para el debate socio-ambiental, pues existen lugares, cosmovisiones y prácticas donde es imposible establecer dicotomías tan plausibles. Grove y Rackhan (2001), por ejemplo, señalan que en el occidente europeo, sobre todo en la cuenca mediterránea, existen espacios alterados por la acción humana a lo largo de la historia, lo que refiere no sólo a cómo los humanos se aprovechan del entorno, sino más bien a la complicidad que se ha adquirido en estos contextos con la naturaleza durante el tiempo, permitiendo vivir en y con ella. ${ }^{4}$ Esa falta de retorno expresa no sólo una carencia en la voluntad de la investigación, sino que atraviesa críticamente la epistemología, la metodología y las técnicas del trabajo etnográfico. Por un lado porque demuestra insuficiencia reflexiva para las condiciones de producción académica (Bourdieu 2003) y por otro porque ejemplifica las formas de hacer periféricas ciertas antropologías (Oliveira 1999; Narotzky 2010).

4 Lo mismo apunta Coca (2010), por ejemplo, al referirse a los "indígenas interiores" andaluces, observando cómo discurso tecno-científico y políticas ambientales chocan con formas de vida históricas vinculadas al medio que se resisten a desaparecer ante una imposición dualista que concibe el entorno "sin gente". 


\section{NOSOTROS TAMBIÉN SOMOS INDÍGENAS:}

\section{ESBOZO DE UNA "ANTROPOLOGÍA DE LA CONVIVENCIA"}

Durante el inicio de mi trabajo de campo con un colectivo de cazadores en el sur occidental de Extremadura (España), me di cuenta de que las proposiciones que venía leyendo sobre el naturalismo no tenían una virtualidad efectiva en la realidad que observaba. ${ }^{5}$ Incluso una práctica social como esta, considerada el epítome de la rapacidad, la depredación o la dominación de la naturaleza en Occidente, no podía explicarse desde dicotomías que dieran sentido a las relaciones entre los cazadores y su entorno.

Enclavados en las estribaciones norte de la Sierra Morena extremeña, una cordillera que atraviesa de este a oeste la Península Ibérica, las poblaciones locales en estos espacios siguen viviendo principalmente de la agricultura y la ganadería. Allí las relaciones exceden las propiamente humanas, siendo necesario valorar las vinculaciones continuadas entre humanos y animales domésticos, de crianza o silvestres, materializadas cada día de diferentes maneras. La actividad de la caza, entonces y como mínimo, tenía que comprenderla como proceso anidado en las interacciones amplias de los grupos campesinos, ya que esta ha sido una actividad complementaria a ese sistema agrosilvoganadero hasta no hace mucho tiempo.

Uno de los primeros días en los que me ofrecí para acompañar a un ganadero a realizar sus tareas diarias entendí el sentido profundo de las relaciones entre humanos y animales en estos contextos. Al llegar a su campo, a unos doce kilómetros por carreteras y caminos de la localidad, el ganadero me indicó que había una vaca tumbada en el suelo mientras que las demás, unas treinta, pastaban dispersas por la finca. No alcancé a verla hasta que él la señaló con el dedo. Observé que efectivamente sobresalía el color del animal sobre el verde de la hierba. Santi, que se llama el ganadero, cambió la tonalidad de la voz y empezó a lamentarse. Yo todavía no sabía qué pasaba, pero cuando llegamos a la altura de la vaca con el todoterreno frenó bruscamente y salió a correr en su dirección. Le seguí bruscamente tropezando por piedras y ramas dispersas por el suelo.

Una vez llegué vi que al lado de la vaca había un becerro, colorado de piel, a diferencia de la novilla blanca. Santi le dio varias vueltas. Al preguntarle qué había pasado el ganadero levantó la cabeza y con ojos medio llorosos me espetó: "se ha muerto la novilla en el parto". Su semblante y la situación, en medio de un bosque de encinas, me estremecieron. Pero Santi sabía que el becerro estaba vivo aunque a mí no me lo pareciese. A pesar de ello, el ganadero arrojó su malestar a través de insultos abstractos al tiempo que golpeaba con los pies pequeños ripios.

5 El trabajo de campo lo comencé en el año 2011, e ininterrumpidamente lo continúo en la actualidad para la realización de mi tesis doctoral, en la localidad sur extremeña de Segura de León. 
Poco después se apartó y se subió en lo alto de una piedra grande para coger cobertura y llamó a su hermano Félix. Le comunicó el fallecimiento de la novilla, la cual me enteré entonces que tenía por nombre Felipa. Cuando Santi terminó la conversación telefónica se vino hacia mí y me ordenó que fuera con el coche al pueblo, a su casa, y le dijera a su mujer que me diera los calostros de cabra que tenía congelados en el frigorífico. ${ }^{6}$ Él me esperaría allí "lavando" al becerro. De la novilla muerta se encargaría su hermano. ${ }^{7}$

Así lo hice y al regresar de nuevo con dos botellas de calostros Santi había cambiado su actitud. Le había puesto nombre al becerro, se llamaría Felipe. Lo montamos limpio en la parte trasera del coche y lo llevamos a la casilla del campo. Calentamos los calostros en una cuba metálica al fuego lento de su anafe, hasta que estuvieran tibios "como para un niño", comentó, y justo después comenzamos a darle de mamar. Felipe bebió casi dos litros. Santi me dijo que ya no se moriría. Le hicimos una cama de paja y allí lo dejamos, en buen cobijo y en un lugar caliente. Ese día hicimos el resto de tareas, sin embargo, Felipa, la novilla fallecida, fue un lamento para Santi todo el día, no sólo por la pérdida material y económica, decía, sino también porque en dos años y medio de vida le había "cogido cariño al animal".

Dos semanas después volví a hablar con Santi para preguntarle sobre el estado de Felipe. Me dijo que fuese yo a visitarlo. En ese período de tiempo el ganadero le había buscado "tres madres postizas", es decir, tres cabras con las ubres lo bastante grandes como para poder dar de mamar a Felipe. Mientras que Santi agarraba por el cuello a las "pobres cabras" - decía él -, había enseñado a su perro Curro a sujetar a Felipe por el rabo para que no se asustara si alguna de las cabras daba patadas (ver figura 1). Así crió al becerro durante un mes, siendo Santi el "padre postizo de Felipe" y las tres cabras las nodrizas, mientras que Curro hacía "de hermano mayor", según la definición del ganadero. Un mes y medio más tarde le pregunté a Santi por Felipe; apenado pero contento al mismo tiempo, me dijo: "lo he vendido para semental porque lo

6 Santi trabaja con una piara de 200 cabras. Vende la leche desde noviembre a junio, periodo de ordeño que él tiene establecido. En octubre nacen los cabritos que se venden en diciembre, para Navidad. De todos los que nacen deja una reposición de hembras para ir sustituyendo las posibles bajas. Las primeras leches de las cabras tras el parto, los calostros, a veces sobran, ya que las cabras tienen ubres muy grandes que generan más leche de la que puedan beber uno o dos cabritos, que son normalmente el número de crías que tienen estos animales. Entonces Santi extrae el sobrante y lo congela para ocasiones como esta donde "algún animal queda huérfano", según sus palabras, por muerte en el parto de la madre. Normalmente estos calostros se administran a vacas, a cabras o a ovejas.

7 Desde hace varios años los animales domésticos muertos han de ser declarados a las aseguradoras para proceder a su incineración previa recogida del animal fallecido en el campo. Posteriormente se lo llevan en camiones herméticos a un centro común donde realizan todos los procesos. Es una nueva normativa de bioseguridad y de sanidad animal que impide las ecorregulaciones que realizaban los animales carroñeros como los buitres o los cuervos, con las cuales estaban más contentos los ganaderos. 


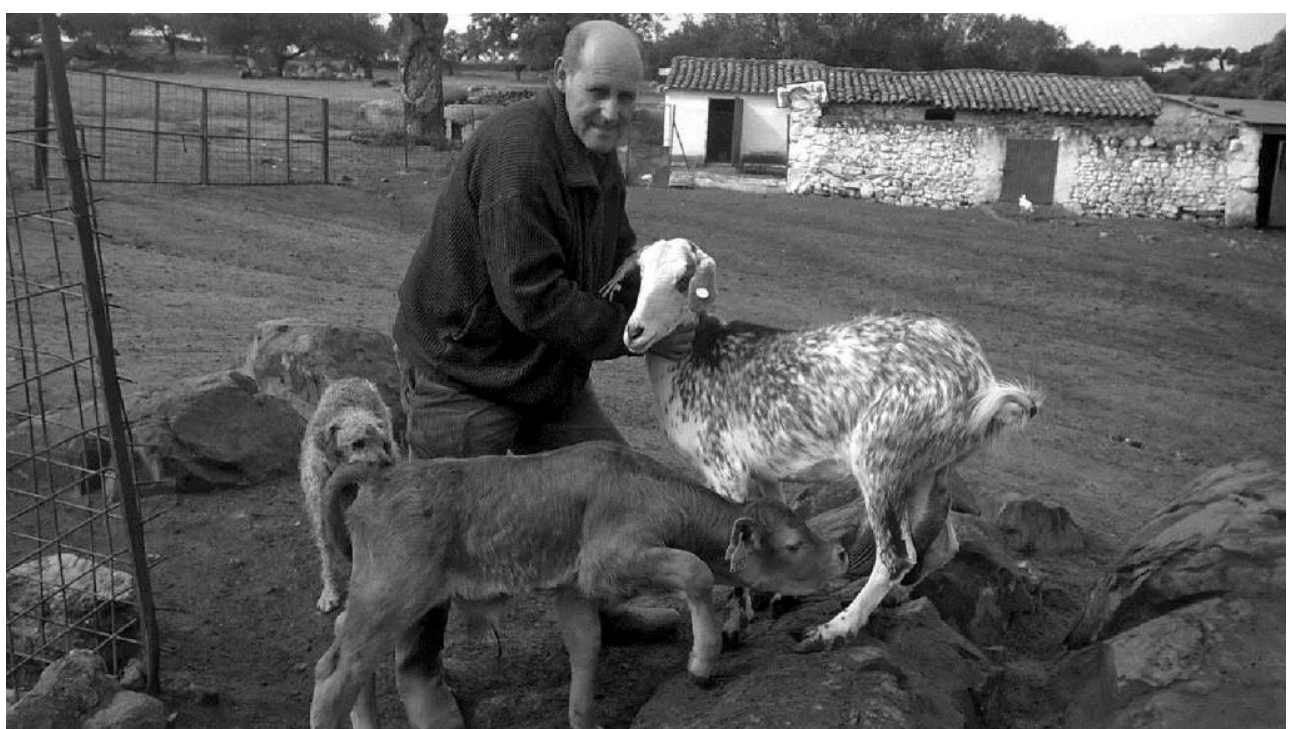

Figura I - Santi sujetando a una de las "madres" de Felipe mientras que Curro, su "hermano mayor", le retiene el rabo para que mame. Foto: Santiago M. Cruzada.

hubiese tenido que mandar al matadero... ya tenemos dos toros... al que se lo he vendido le he dicho que se llama Felipe".

Este episodio extraído de mi trabajo de campo servirá para esbozar una "antropología de la convivencia" que haga ampliar el alcance de la etnografía hacia ámbitos que superen los límites que impone el naturalismo, entendiendo las relaciones entre sociedad y naturaleza de manera enmarañada para contextos occidentales como el que estoy refiriendo. Si bien las perspectivas que se basan en el naturalismo como fundamento epistemológico han creado un marco idóneo para el estudio socioecológico, sus generalidades se agotan en los múltiples casos en los que el naturalismo no opera como se le define. No obstante, extraeré de ellas ideas que apoyen las mías para cuestionar, más si cabe, la efectividad del modelo naturalista en su circunscripción estereotipada contextual.

Desde el punto de vista de la representación de las relaciones, es decir, la representación metafórica y la objetivación conceptual de la naturaleza (Rival 2004: 97-98), Santi estaría dentro de una comunidad conformada por diferentes seres que habitan el entorno (Descola 2004: 32). En su día a día con los animales no distingue sobre criterios específicos que lo diferencien del resto de individuos, pues aquellos aparecen como entidades caracterizadas no por sus cualidades como animales, sino por caracteres abstractos como los utilizados para las personas. Habla de la personalidad de los animales, de su temperamento, de sus problemas, de su historia individual, de su parentesco con otros animales o, por ejemplo, de su modo de comer. Su interacción hace borrosas las 
fronteras entre seres en un contexto donde es necesaria la cooperación para la vida. Si efectivamente en última instancia Santi vive de ello, los animales también viven de Santi, por lo que la idea de un aprovechamiento de la naturaleza (Descola 2001: 1 10-112) no tiene sentido porque la misma idea de naturaleza queda agotada.

La "objetificación" de su cotidianeidad la realiza en términos igualitarios en relación a las dimensiones básicas de la vida social (Descola 2012), muy influenciadas por las categorías de parentesco como esferas de unas relaciones cotidianas íntimas y de proximidad. Así, por ejemplo, dotar de nombre social a los animales, en este caso Felipa y su cría Felipe (aunque esto es extensivo a otros animales), orienta a pensar que las relaciones sociales de parentesco humano tienen un sentido extendido, y el hecho de que le pusiera a la cría el nombre de la madre nos muestra la voluntad por hacer duraderas esas relaciones, prolongadas en este caso de forma matrilineal. ${ }^{8}$

La animalidad y la humanidad quedarían aquí redefinidas ya que el sujeto queda ampliado y supera al objeto, siendo el ser de cada humano o animal el que juega un papel importante en las relaciones (Souza 2013), lo que se enfrenta a nuestras oposiciones históricas igualmente. El hecho de "calentar los calostros como para un niño" pone de manifiesto la cualidad relativa de perspectivas. Es una relación construida que toma como base las propiedades corporales humanas y la experiencia del cuidado infantil para traspasarlas al cuerpo animal. Se resalta, por tanto, el carácter social que se produce entre la serie humana y no humana (Castro 2004: 46).

Efectivamente, la investigación aquí tampoco puede estar centrada en los individuos entendidos como pasivos y autónomos. Más bien ha de enfocarse a las personas completas actuando dentro de contextos particulares (Descola y Pálsson 2001: 17), comprendiendo la diversidad de influencias recíprocas en el vivir cotidiano. Lo que a primera vista pudiese estar separado desde una perspectiva naturalista queda anexionado, pues las vinculaciones sociales abarcan más que las propias relaciones humanas (Escobar 2000: 119) en términos de simetrías ante las circunstancias de la vida que son compartidas (Latour 2008).

En el ejemplo que propongo el sistema de parentesco opera sobre los modos de relacionarse, lo que acarrea obligaciones y deberes con/para otros seres con los que se comparten vivencias, que incluyen al mismo tiempo pautas de clasificación y modos de identificación (Descola 2001). De tal modo se conforma un mundo ampliado en un contexto involucrado que viene definido por la redistribución de "compromisos" dependiendo de la especificidad necesaria para cada acontecimiento convivido, pero con una base socialmente reconocida.

8 En un sentido similar se posiciona Blanchard (2015) al observar que entre personas sin hogar que poseen perros el nombre asignado al animal es fundamental no sólo para guardar el vínculo afectivo, sino que arroja luz sobre las historias de vida de sus propietarios. 
En este caso vendrían establecidas desde una idea de parentesco reducido al núcleo familiar inmediato: un padre (Santi), tres madres (las cabras), un hermano mayor (el perro) y Felipe, el ternero que tendría el perfil de un bebé lactante. Que el padre y las tres madres sean expresados en términos "postizos" refuerza, pero no suprime, la relación original sobre la que se basa. ${ }^{9}$

En esa diversificación de responsabilidades con otros seres el lenguaje adquiere gran significación. A través de patrones lingüísticos se dota de agencia a los seres interactuantes desde un punto de vista intersubjetivo y representacional (Ingold 1992; Kohn 2007). Esto se ve materializado en las pautas comunicativas que se establecen en la cotidianeidad. Pero no todos los individuos aparecen involucrados ni en la misma medida. ${ }^{10} \mathrm{Al}$ volver por segunda vez para comprobar el estado de Felipe, Santi llamó a las tres cabras con el estilo que se utiliza en estos contextos. Una locución abreviada ("mina, mina, mina") seguida de un silbido fueron suficientes para que las tres madres, sabiendo lo que tenían que hacer, se apartaran de la piara de doscientas cabras y se acercaran al ganadero. Por otro lado llamó a Curro, que estaba tumbado debajo del todoterreno, con un peculiar "Curro ivamos!". Por último llamó a Felipe con una repetición sonora: "toma, pué, Felipe, toma, pué". ${ }^{11}$ El becerro se acercó a ellos y comenzó a mamar de la cabra mientras que Curro le sujetaba el rabo y Santi sostenía la cabeza de la cabra. Por el llano de la casa pululaban gatos y

9 Lo refuerza porque el animal "quedó huérfano", como dijo Santi, lo que confiere una relación de parentesco mayor no sólo con el becerro sin madre, sino con la madre misma. Hay que matizar, no obstante, que si bien términos como madre o padre "postizo" son utilizados comúnmente por ganaderos del pueblo ante casos de orfandad animal, que Santi utilizase "hermano mayor" para denominar al perro pudo ser una prolongación nominativa del parentesco humano por la colaboración de este animal en el cuidado diario del becerro. Govindrajan (2015: 505) sostiene, en un caso particular, que el parentesco entre humanos y animales está sustentado por las prácticas diarias de cuidado, atención y sujeción sobre los animales, donde se crea un reino afectivo, relacional y de lenguaje encarnado entre animales humanos y no humanos que permiten una serie de compromisos y reconocimientos mutuos.

10 Un ejemplo de la intensidad y la involucración es el nombre que se le pone a los animales más cercanos. En ese sentido se les otorga un nombre humano que, la mayoría de las veces, tiene su evocación en el grupo social del ganadero. Este nombre, como vimos, es transmitido generacionalmente entre los miembros de la especie. Por ejemplo, Curro se llama así porque su tatarabuelo fue dado a la familia de Santi por un ganadero llamado Curro. Otras veces los nombres están asignados por la semejanza del animal al humano, sobre todo cuando aquellos tienen alguna disfunción corporal.

Il Tanto "mina, mina, mina" como "pué, toma, pué" son locuciones que proceden de una base onomatopéyica, es decir, es una representación del sonido que hacen los animales. Este sonido es interpretado por el ganadero de diferentes maneras, de tal forma que cuando una cabra come hace un sonido parecido al que es utilizado por el ganadero para llamarlas. Las vacas, específicamente las crías, realizan un sonido cuando tienen hambre que es el que utiliza de base el ganadero. Sin embargo, en esta representación onomatopéyica se proyectan categorías sociales como la edad o el género. Así a los machos cabríos se les llama con "mino" y a las crías se les reclama con "mini", distinguiendo con léxico masculino el primero y con una semántica "delicada" en referencia a la edad el segundo, respondiendo cada animal, efectivamente, a esa llamada sin mezclarse. 
gallinas, los cuales iban "a su aire", como si ese asunto no tuviese "nada que ver con ellos", dijo Santi.

La representación de la relación con los animales a través de un léxico del parentesco reducido al núcleo familiar inmediato encuentra valor tanto por lo que son en sí mismas como por lo que se les atribuye significativamente, y la comunicación que el ganadero establece con los animales queda al servicio de la construcción cultural de un mundo ampliado lleno de sentidos que se desarrollan con la experiencia. Pero la comunicación verbal que se produce entre especies actúa como imperativo lingüístico que hace explícito los procesos de difuminación ontológica entre ellos, incorporando modalidades comunicativas de cada especie en el lenguaje humano, o lo que es lo mismo, enredos a través de códigos lingüísticos trans-especies (Kohn 2007: 14).

Los animales en este caso no están solamente para ser utilizados para el interés propio humano, para su explotación, ni tan siquiera se presentan como facilitadores de la interpretación humana (Ingold 1992), sino que están, como sostiene Haraway (2003: 5), para "vivir con”. En el caso propuesto, los animales representan una intencionalidad a través de señales corporales que funcionan como indicadores comunicativos en una ecología que podría llamarse "de los seres" (Kohn 2007: 4) redefinida ahora para contextos occidentales. ${ }^{12}$ Esto no elimina que existan, de alguna manera, en los mecanismos necesarios para el control y el cuidado ganadero, relaciones de dominación y sumisión entre humanos y animales (Ellen 1999), que sin embargo son inherentes a la sociabilidad de cada especie doméstica al elegir a figuras alfas de liderazgo en las piaras. En definitiva, sugiero que el significado, de acuerdo con Kohn (2007: 5) o Goodwin (2007: 100), no es exclusivo de los seres humanos. Estos, al concebirse como entidades que existen junto a otros y entre otros, más que relacionarse individualmente con el entorno, lo integran (Ingold 2000).

Esto me lleva a analizar las relaciones desde el punto de vista de la percepción, o lo que es lo mismo, a reconsiderar para contextos occidentales los términos de la acción y la experiencia práctica de los humanos en el entorno (Rival 2004: 97). El mundo perceptivo y la experiencia vivida son inseparables de la representación humana y pueden ser comprendidos como un adiestramiento involucrado en el medio ambiente (Ingold 2001: 55-57). Así por ejemplo, en nuestro caso concreto, Santi localizó la vaca muerta a gran distancia. Felipa yacía sobresaliente entre una gran extensión de hierba verde, pero la diversidad cromática que acompañaba esa imagen impidieron que yo me fijara en eso.

12 Por ejemplo, las cabras mueven las orejas de un lado a otro durante tres o cuatro veces al mismo tiempo que "estornudan" sacando la lengua hacia sus orificios nasales. El perro mueve el rabo constantemente y se posiciona detrás de Santi. El becerro Felipe brinca y mueve el rabo al mismo tiempo que muge en señal de felicidad, según interpreta Santi. 
Santi me aseguró que le "saltó a la vista" aquella imagen por ser anómala en su día a día. El entorno había cambiado y Santi lo percibió de inmediato.

Asimismo, cuando bajó del todoterreno, Santi corrió hacia la vaca como si lo estuviese haciendo sobre una superficie lisa. Yo, sin embargo, tropecé muchas veces con piedras y ramas que había en el trayecto. Como dice Escobar (2000: 121), los seres humanos se encuentran inmersos en los entornos a través de actos prácticos localizados que definen diferentes arraigos contextuales producidos en el seno de una cultura específica. Santi tenía incorporada una forma de pisar que, incluso trabándose con algún elemento del entorno, no lo desestabilizaban. El cuerpo es el modo de ser y de estar en el mundo, no es una posición como objeto, sino una situación como sujeto (Almeida 1996: $11-12) \cdot{ }^{13}$

Si bien percepción y representación (a la cual he incluido elementos comunicativos) son la base analítica para la mayoría de estudios de relaciones socioecológicas, entiendo que para comprender de manera definitiva las relaciones ambientales que se producen en Occidente es necesario conjugar otras variables que abarquen las ambigüedades y paradojas que en estos contextos operan. Una teoría social ha de explorar lo emotivo como campo de la vida social que no está separado de la vida perceptual, estando en interrelación con la realidad representada y la práctica social extensa (Csordas 1990). Las emociones, los valores, los sentimientos y los afectos se integran en un marco amplio de convivencia, son aprendidos, compartidos y operan en cada representación o percepción del mundo.

En otras palabras, examinarlos es necesario porque muchas veces definen las rutas individuales de acción y pensamiento que explicitan de manera profunda las interconexiones que se producen entre los seres humanos y sus entornos, es decir, redefinen las dimensiones propias de la experiencia vivida y del acto de conocer (Surrallés 2005). Sin embargo, entender las emociones como signos no es otra cosa que interpretarlas como hechos objetivos que guían metafóricamente una clasificación esencialista de creencias y prácticas, por lo que conviene sustituir esta unidad mínima por el conjunto de significantes y actos que muestran un cuerpo afectivo (Surrallés 2005: 5-6). Es conveniente situar las emociones en el campo de lo relacional, alejadas de los estados absolutos (Le Breton 1999).

Entonces, si entendemos el campo emotivo como experiencia socialmente compartida, en el caso que propongo las experiencias sensitivas no humanas ya no estarían fuera de la capacidad explicativa en las etnografías (Latour 2014).

13 Del mismo modo, cuando Santi quiso llamar a su hermano Félix para comunicarle que Felipa había fallecido en el parto, vio que su teléfono móvil no tenía cobertura. Inmediatamente se desplazó hacia una piedra grande y elevada donde sabía que cogería línea. Fue un acto específico y literalmente su cuerpo hizo las funciones de antena telefónica, funcionando, en el doble sentido, como transmisor y receptor de información (Douglas 2004). 
Los humanos no pueden ser descritos ajenos a los mundos que ellos mismos representan (Kohn 2013: 9). Es por ello por lo que la emotividad ha de ser entendida como un acontecimiento apoyado en un sistema de sentidos y valores comunes, o lo que es lo mismo, basados en una cultura afectiva y en sentimientos compartidos (Le Breton 1999: 22). En este caso los sentimientos son compartidos de manera inter-específica, es decir, entre los diferentes seres que habitan y conviven en un mismo entorno.

Durante el proceso en el que todos los animales hacían una coreografía para alimentar a Felipe, Santi acariciaba a cada uno de ellos. Estos le respondían con señales corporales interpretadas por el ganadero como alegría para el perro y el becerro, y como paciencia para la cabra. ${ }^{14}$ Así, el vínculo afectivo que él mantiene con los animales, y los animales con él, adquiere una forma homeostática que mantiene dentro de un universo de sentidos interdependencia entre los diferentes actores (Le Breton 1999: 99). ${ }^{15}$ El hecho de que cierto tipo de animales utilicen signos interpretables para las emociones y los afectos ante circunstancias concretas no sólo muestra que existe mundo más allá de los sistemas humanos de significado (Khon 2013), sino que pone de manifiesto cómo ese significado se ha ido construyendo de manera compartida entre humanos y animales a partir de una convivencia íntima a lo largo del tiempo.

Los valores asignados a los individuos en esa circunstancia pivotan sobre los atributos sociales de una sociedad local ensimismada como en la que se encuentra Santi (Catani, Amaya y Díaz 2001), donde la lealtad, el apoyo mutuo, la correspondencia o incluso el consentimiento son compartidos tanto por humanos como por animales. De ahí la razón de ser del comportamiento animal, el cual se desea que sea social. De lo contrario perdería su condición pasando al plano de la pura animalidad, separada de lo humano. Aquí se considera el respeto y la confianza como el desempeño transcendental en las relaciones humanas con los animales (Haraway 2003: 39). De este modo se impone una clasificación social y de valor al mundo de los animales, y los términos que se usan para definir el comportamiento colectivo de humanos se traslada al mundo no humano (Ellen 2001: 136). Al darle sustento, apoyo y alimento a Felipe, tanto Curro el perro, como las tres madres postizas y Santi, se unen como parte de un mismo campo afectivo que va más allá de sus límites como especies, esto es, un efímero "yo" distribuido entre los diferentes cuerpos (Kohn 2007: 17).

Sin embargo, al preguntarle a Santi por Felipe días más tarde y decirme que lo había vendido para semental se produjo una súbita duda: si tanto lo

14 Cuando la cabra movía la cabeza o la pata Santi decía "pobre cabrita, ya falta poco", intentando tranquilizarla con la voz pero también con caricias. Según Santi, las embestidas del becerro mamando causaban dolor en el animal, de ahí que se refiriera a la "paciencia" de la cabra.

15 En el momento que Santi vio a la vaca muerta se lamentó profundamente pues le "había cogido cariño”. Luego la ira le sobrevino y lo expresó dando patadas a piedras en señal de enojo. 
había cuidado, tanto lo quería y tanto se lamentó por su madre, ¿cómo es que lo había vendido? Su contestación fue rotunda: "ya tenía otros sementales" y, antes de enviarlo al matadero, había preferido buscar un comprador que lo cuidara para reproductor. La economía campesina se interpuso a los sentimientos, sin embargo, Santi jugó con ambos factores, pues al mismo tiempo que lo había vendido garantizó la continuidad de la vida del animal. El discurso que separa a humanos y animales en estos contextos pierde su sentido cuando la decisión humana acerca de la continuidad de la vida de un animal crea cargos de conciencia y establece remordimientos. Humanos y animales, en definitiva, se unen como parte de un mismo campo que va más allá de sus límites como especies en procesos de "personalidad flexible" (Shir-Vertesh 2012: 421).

Estos cuatro bloques analíticos no tendrían alcance sin atravesarles variables tangenciales que, de manera inevitable, operan sobre las relaciones entre las diferentes especies que habitan el entorno. La edad o el género juegan un papel fundamental no sólo en el plano humano, sino también en el ámbito animal, como comprobamos en las interacciones comunicativas. Así, es necesario no olvidar cuestiones que tienen que ver con el posicionamiento de los actores, ya sea desde el plano de las clases sociales para los humanos como desde el plano de especies para animales. Como vimos, no todos los animales se encuentran involucrados ni en la misma medida en la convivencia socioecológica, aspectos que convenientemente sigo utilizando en el estudio de la caza.

\section{CONCLUSIONES}

He intentado llevar lo más lejos posible un análisis que permita comprender la efectividad de las diferentes formas de relación que pueden producirse en entornos occidentales donde, a pesar de las propuestas proyectivas estereotipadas sobre estos contextos, se pueden extraer ideas claras de seres relacionales tomando conciencia no sólo de su posición en el mundo, sino también dándole la importancia necesaria a cómo escuchan, cómo se comportan o cómo sienten (Bird-David 1999: s72). La investigación sobre las vinculaciones inter-especies sitúa a los animales como parte importante, e indisociable, de las realidades humanas (Knight 2005: 1), lo que al mismo tiempo permite repensar contextos periféricos y asignaciones epistemológicas parciales.

Las limitaciones de un naturalismo impuesto excluyen a Occidente, sin matices, de una reflexión monista de las relaciones entre la sociedad y la naturaleza. Las teorías que se sustentan en este modelo caen en una doble forma de homogeneización: definen el contexto occidental asignándole categorías dicotómicas tanto a la sociedad en general como al conocimiento que produce académicamente y representan en su investigaciones sociedades esencializadas donde se excluyen variables como la edad, el género o la influencia del campo emotivo sobre las prácticas y las representaciones. 
El naturalismo, tal y como es definido, presenta vulnerabilidades en contextos occidentales específicos donde la naturaleza no queda ajena a la voluntad humana. Una antropología centrada en la convivencia debe intentar mostrar cómo ciertas prácticas, situaciones, actividades o espacios occidentales están conformados a partir de la idea de mundos ampliados que incluyen dentro de contextos involucrados a diferentes seres que habitan el entorno, superando las relaciones sociales humanas y alcanzando un tejido interactivo de dilatado espectro. Este texto ha pretendido ser una muestra de ello y una llamada de atención para un tipo de antropología centrada en recorrer altas esferas académicas antes que en transitar las realidades que deconstruye.

\section{BIBLIOGRAFÍA}

ALMEIDA, Miguel Vale de, 1996, "Corpo presente", en Miguel Vale de Almeida (coord.), Corpo Presente: Treze Reflexões Antropológicas sobre o Corpo. Oeiras, Celta Editora, 1-22.

BARTOLOMÉ, Miguel, 2014, "El regreso de la barbarie: una crítica etnográfica a las ontologías 'premodernas'”, Publicar en Antropología y Ciencias Sociales, XII (16): 9-33, disponible en < http://ppct.caicyt.gov.ar/index.php/publicar/article/view/5588 > (última consulta en enero 2017).

BESSIRE, Lucas, y David BOND, 2014, "Ontological anthropology and the deferral of critique”, American Ethnologist, 41 (3): 440-456.

BIRD-DAVID, Nurit, 1999, “'Animism' revisited: personhood, environment, and relational epistemology”, Current Anthropology, 40 (S1): s67-s91.

BLANCHARD, Christophe, 2015, "Homeless people with dogs: what can be learned from the animals' names?", Anthropozoologica, 50 (2): 99-107.

BLASER, Mario, 2009, "Political ontology: cultural studies without 'cultures'?", Cultural Studies, 23 (5): 873-896.

BOURDIEU, Pierre, 2003, El Oficio de Científico: Ciencia de la Ciencia y Reflexividad. Barcelona, Anagrama.

CANDEA, Matei, 2011 , “Endo/Exo”, Common Knowledge, 17 (1): 146-150.

CANDEA, Matei, y Lys ALCAYNA-STEVENS, 2012, "Internal others: ethnographies of naturalism”, Cambridge Anthropology, 30 (2): 36-47.

CARRITHERS, Michael, et al., 2010, "Ontology is just another word for culture: motion tabled at the 2008 meeting of the Group for Debates in Anthropological Theory, University of Manchester", Critique of Anthropology, 30: 152-200.

CASTRO, Eduardo Viveiros de, 2004, "Perspectivismo y multinaturalismo en la América indígena", en Alexander Surrallés y Pedro García (coords.), Tierra Adentro: Territorio Indígena y Percepción del Entorno. Copenhague, IWGIA, 37-79. 
CASTRO, Eduardo Viveiros de, 2010, Metafísicas Caníbales: Líneas de Antropología Postestructural. Buenos Aires, Katz.

CATANI, Mauricio, Santiago AMAYA, y Antonio Luis DÍAZ, 2001, Comer en Tentudía: Aproximación Etnográfica a la Comida y a los Hábitos de Vida de las Gentes de la Comarca de Tentudía en los Últimos Setenta Años. Monesterio, CEDECO Tentudía.

CITRO, Silvia, y Mariana GÓMEZ, 2013, "Perspectivismo, fenomenología cultural y etnografías poscoloniales: intervenciones en un diálogo sobre las corporalidades", Espaço Ameríndio, 7 (1): 253-286, disponible en < http://seer.ufrgs.br/index.php/EspacoAmerin dio/article/view/36990/25966 > (última consulta en enero 2017).

COCA, Agustín, 2010, "Políticas ambientales y marginación campera: las nuevas 'reservas indígenas' en la Andalucía del siglo XXI”, en Marta Soler y Carmen Guerrero (coords.), Patrimonio Cultural en la Nueva Ruralidad Andaluza. Sevilla, Junta de Andalucía, col. Cuadernos PH, n. ${ }^{\circ}$ 26, $111-125$.

COSTA, Luiz, y Carlos FAUSTO, 2010, “The return of the animists: recent studies of Amazonian ontologies”, Religion and Society: Advances in Research, 1 (1): 89-109.

CROLL, Elisabeth, y David PARKIN, 1992, "Cultural understandings of the environment", en Elisabeth Croll y David Parkin (coords.), Bush Base: Forest Farm. Culture, Environment and Development. Londres, Routledge, 11-38.

CSORDAS, Thomas, 1990, "Embodiment as a paradigm for anthropology", Ethos, 18 (1), 5-47.

DE LA CADENA, Marisol, 2014, “The politics of modern politics meets ethnographies of excess through ontological openings", Cultural Anthropology Online, disponible en $<$ http://www.culanth.org/fieldsights/47 l -the-politics-of-modern-politics-meets-ethnographies-of-excess-through-ontological-openings $>$ (última consulta en enero 2017).

DESCOLA, Philippe, 2001, "Construyendo naturalezas: ecología simbólica y práctica social”, en Philippe Descola y Gísli Pálsson (coords.), Naturaleza y Sociedad: Perspectivas Antropológicas. México, Siglo XXI, 101-123.

DESCOLA, Philippe, 2004, "Las cosmologías indígenas de la Amazonía", en Alexander Surrallés y Pedro García (coords.), Tierra Adentro: Territorio Indígena y Percepción del Entorno. Copenhague, IWGIA, 25-36.

DESCOLA, Philippe, 2012, Más Allá de Naturaleza y Cultura. Buenos Aires, Amorrortu Editores.

DESCOLA, Philippe, y Gísli PÁlSSON, 2001, “Introducción”, en Philippe Descola y Gísli Pálsson (coords.), Naturaleza y Sociedad: Perspectivas Antropológicas. México, Siglo XXI, 11-35.

DIÉGUEZ LUCENA, Antonio, 2014, “Delimitación y defensa del naturalismo metodológico (en la ciencia y en la filosofía)”, en Raúl Gutiérrez-Lombardo y José Sanmartín (orgs.), La Filosofía desde la Ciencia. México, DF, Centro de Estudios Filosóficos, Políticos y Sociales Vicente Lombardo Toledano, 21-49.

DOUGLAS, Mary, 2004, Natural Symbols: Explorations in Cosmology. Londres, Routledge.

ELLEN, Roy, 1999, "Categories of animality and canine abuse: exploring contradictions in Nuaulu social relationships with dogs", Anthropos, 94 (1-3): 57-68.

ELLEN, Roy, 2001, "La geometría cognitiva de la naturaleza: un enfoque contextual", en Philippe Descola y Gísli Pálsson (coords.), Naturaleza y Sociedad: Perspectivas Antropológicas. México, Siglo XXI, 124-146. 
ESCOBAR, Arturo, 2000, "El lugar de la naturaleza y la naturaleza del lugar: ¿globalización o postdesarrollo?”, en Andreu Viola (comp.), Antropología del Desarrollo. Barcelona, Paidós, 113-144.

ESCOBAR, Arturo, 2011 , "Epistemologías de la naturaleza y colonialidad de la naturaleza: variedades de realismo y constructivismo", en Leonardo Montenegro (coord.), Cultura y Naturaleza: Aproximaciones a Propósito del Bicentenario de la Independencia de Colombia. Bogotá, Jardín Botánico de Bogotá, José Celestino Mutis, 49-74.

FISCHER, Michael, 2014, "The lightness of existence and the origami of 'French' anthropology: Latour, Descola, Viveiros de Castro, Meillassoux, and their so-called ontological turn", HAU: Journal of Ethnographic Theory, 4 (1): 33 1-355.

GLACKEN, Clarence, 1996, Huellas en la Playa de Rodas: Naturaleza y Cultura en el Pensamiento Occidental desde la Antigüedad hasta Finales del Siglo XVII. Barcelona, Ediciones del Serbal. GOODWIN, Brian, 2007, Nature's Due: Healing Our Fragmented Culture. Edimburgo, Floris Books.

GOVINDRAJAN, Radhika, 2015, “'The goat that died for family': animal sacrifice and inerspecies kinship in India's Central Himalayas", American Ethnologist, 42 (3): 504$-519$.

GRABER, David, 2015, "Radical alterity is just another way of saying 'reality': a replay to Eduardo Viveiros de Castro", HAU: Journal of Ethnographic Theory, 5 (2): 1-41.

GRAY, Andrew, 1996, The Arakmbut of Amazonian Peru, vol. I: Mythology, Spirituality, and History in an Amazonian Community. Providence y Oxford, Berghahn Books.

GROVE, Alafred, y Oliver RACKHAM, 2001, The Nature of Mediterranean Europe: An Ecological History. New Haven, Yale University Press.

GUILlO, Dominique, y Peter HAMILTON, 2015, "What is the place of animals in the social sciences? The limits to the recent rehabilitation of animal agency", Revue Française de Sociologie (English Edition), 56 (1): 116-141.

HAGE, Ghassan, 2014, "Critical anthropology as a permanent state of first contact", Cultural Anthropology Online, disponible en < http://www.culanth.org/fieldsights/473-critical-anthropology-as-a-permanent-state-of-first-contact $>$ (última consulta en enero 2017).

HARAWAY, Donna, 2003, The Companion Species Manifesto: Dogs, People, and Significant Otherness. Chicago, Prickly Paradigm Press.

HENARE, Amiria, Martin HOLBRAAD, y Sari WASTELL, 2007, Thinking through Things: Theorising Artefacts Ethnographically. Londres, Routledge.

INGOLD, Tim, 1991, "Becoming persons: consciousness and sociality in human evolution", Cultural Dynamics, 4 (3): 355-387.

INGOLD, Tim, 1992, "Culture and the perception of the environment", en Elisabeth Croll y David Parkin (coords.), Bush Base: Forest Farm. Culture, Environment and Development. Londres, Routledge, 39-56.

INGOLD, Tim, 1994, "Introducción”, en Tim Ingold (coord.), What Is an Animal? Londres y Nueva York, Routledge, 1-16.

INGOLD, Tim, 2000, The Perception of the Environment: Essays in Livelihood, Dwelling and Skill. Londres, Routledge.

INGOLD, Tim, 2001, "El forrajero óptimo y el hombre económico", en Philippe Descola y Gísli Pálsson (coords.), Naturaleza y Sociedad: Perspectivas Antropológicas. México, Siglo XXI, 37-59. 
INGOLD, Tim, 2008, “Tres en uno: cómo disolver las distinciones entre cuerpo, mente y cultura”, en Tomás Sánchez Criado (coord.), Tecnogénesis: La Construcción Técnica de las Ecologías Humanas, vol. 2. Madrid, Antropólogos Iberoamericanos en Red, 1-34.

INGOLD, Tim, 2016, "A naturalist abroad in the museum of ontology: Philippe Descola's beyond nature and culture", Anthropological Forum, 26 (3): 301-320.

KNIGHT, John, 2005, “Introduction”, en John Knight (coord.), Animals in Person: Cultural Perspectives on Human-Animal Intimacies. Oxford, Berg, 1-13.

KOHN, Eduardo, 2007, "How dogs dream: Amazonian natures and the politics of transpecies engagement", American Ethnologist, 34 (1): 3-24.

KOHN, Eduardo, 2013, How Forests Think: Toward an Anthropology beyond the Human. Berkeley, University of California Press.

KOHN, Eduardo, 2015, "Anthropology of ontologies", Annual Review of Anthropology, 44: $311-327$.

LATOUR, Bruno, 2007, Nunca Fuimos Modernos: Ensayo de Antropología Simétrica. Buenos Aires, Siglo XXI.

LATOUR, Bruno, 2008, Reensamblar lo Social: Una Introducción a la Teoría del Actor Red. Buenos Aires, Manantial.

LATOUR, Bruno, 2013, An Inquiry into Modes of Existence: An Anthropology of the Moderns. Cambridge, MA, Harvard University Press.

LATOUR, Bruno, 2014, "On selves, forms, and forces", HAU: Journal of Ethnographic Theory, $4(2): 1-6$.

LE BRETON, David, 1999, Las Pasiones Ordinarias: Antropología de las Emociones. Buenos Aires, Ediciones Nueva Visión.

MARCUS, George, y Michael FISCHER, 2000, La Antropología como Crítica Cultural: Un Momento Experimental en las Ciencias Humanas. Buenos Aires, Amorrortu.

MORALES INGA, Sergio, 2014, "Sobre objetos que vuelan en el cosmos: crítica al perspectivismo amerindio de Eduardo Viveiros de Castro", Patio de Sociales, disponible en < https://www.researchgate.net/publication/292784604_Sobre_objetos_que_flotan_en _el_cosmos_Critica_al_perspectivismo_amerindio_de_Eduardo_Viveiros_de_Castro_ Parte_II_2016> (última consulta en enero 2017).

NAROTZKY, Susana, 2010, "Las antropologías hegemónicas y las antropologías del sur: el caso de España”, Antípoda: Revista de Antropología y Arqueología, 1 1: 241-258.

OLIVEIRA, Roberto Cardoso de, 1999, "Peripheral anthropologies 'versus' central anthropologies", Journal of Latin American and Caribbean Anthropology, 4 (2): 10-31.

PAZOS, Álvaro, 2007, "Alexandre Surrallés y Pedro García, Tierra Adentro: Territorio Indígena y Percepción del Entorno", AIBR: Revista de Antropología Iberoamericana, 2 (2): 369. -377 .

PEDERSEN, Morten, 2011, Not Quite Shamans: Spirits Worlds and Political Lives in Northern Mongolia. Ithaca, NY, Cornell University Press.

RAMOS, Alcida, 2012, "The politics of perspectivism”, Annual Review of Anthropology, 41: 481-494.

REYNOSO, Carlos, 2015, Crítica de la Antropología Perspectivista: Viveiros de Castro, Philippe Descola, Bruno Latour. Buenos Aires, Editorial SB.

RIVAL, Laura, 2004, "El crecimiento de las familias y de los árboles: la percepción del bosque de los Huaorani”, en Alexander Surrallés y Pedro García (coords.), Tierra Adentro: Territorio Indígena y Percepción del Entorno. Copenhague, IWGIA, 97-120. 
SAHLINS, Marshall, 201 1, La Ilusión Occidental de la Naturaleza Humana. México, DF, Fondo de Cultura Económica.

SAHLINS, Marshall, 2014, "On the ontological scheme of Beyond Nature and Culture", HAU: Journal of Ethnographic Theory, 4 (1): 281-290.

SALMOND, Amiria, 2014, "Transforming translations (part 2): addressing ontological alterity", HAU: Journal of Ethnographic Theory, 4 (1): 155-187.

SÁNCHEZ-CRIADO, Tomás, 2009, "Tim Ingold, The Perception of the Environment: Essays in Livelihood, Dwelling and Skill", AIBR: Revista de Antropología Iberoamericana, 4 (1): 142-158.

SHIR-VERTESH, Dafna, 2012, “'Flexible personhood': loving animals as family members in Israel”, American Anthropologist, 114 (3): 420-432.

SOUZA, Iara Maria de Almeida, 2013, "Vidas experimentais: humanos e roedores no laboratório”, Etnográfica, 17 (2): 241-268.

STRATHERN, Marilyn, 2004, "The whole person and its artifacts", Annual Review of Anthropology, 33: 1-19.

SURRALLÉS, Alexandre, 2005, "Afectividad y epistemología de las ciencias humanas", AIBR: Revista de Antropología Iberoamericana, número especial: 1-16.

VIGH, Henrik, y David SAUSDAL, 2014, "From essence back to existence: anthropology beyond the ontological turn", Anthropological Theory, 14 (1): 49-73. 